\title{
Retroperitoneal oblique corridor to the L2-S1 intervertebral discs: an MRI study
}

\author{
Diana M. Molinares, MD, Timothy T. Davis, MD, and Daniel A. Fung, MD \\ Orthopedic Pain Specialists, Santa Monica, California
}

OBJECTIVE The purpose of this study was to analyze MR images of the lumbar spine and document: 1) the oblique corridor at each lumbar disc level between the psoas muscle and the great vessels, and 2) oblique access to the L5-S1 disc space. Access to the lumbar spine without disruption of the psoas muscle could translate into decreased frequency of postoperative neurological complications observed after a transpsoas approach. The authors investigated the retroperitoneal oblique corridor of L2-S1 as a means of surgical access to the intervertebral discs. This oblique approach avoids the psoas muscle and is a safe and potentially superior alternative to the lateral transpsoas approach used by many surgeons.

METHODS One hundred thirty-three MRI studies performed between May 4, 2012, and February 27, 2013, were randomly selected from the authors' database. Thirty-three MR images were excluded due to technical issues or altered lumbar anatomy due to previous spine surgery. The oblique corridor was defined as the distance between the left lateral border of the aorta (or iliac artery) and the anterior medial border of the psoas. The L5-S1 oblique corridor was defined transversely from the midsagittal line of the inferior endplate of $L-5$ to the medial border of the left common iliac vessel (axial view) and vertically to the first vascular structure that crossed midline (sagittal view).

RESULTS The oblique corridor measurements to the L2-5 discs have the following mean distances: $L 2-3=16.04 \mathrm{~mm}$, $\mathrm{L} 3-4=14.21 \mathrm{~mm}$, and L4-5 = $10.28 \mathrm{~mm}$. The L5-S1 corridor mean distance was $10 \mathrm{~mm}$ between midline and left common iliac vessel, and $10.13 \mathrm{~mm}$ from the first midline vessel to the inferior endplate of $\mathrm{L}-5$. The bifurcation of the aorta and confluence of the vena cava were also analyzed in this study. The aortic bifurcation was found at the L-3 vertebral body in $2 \%$ of the MR images, at the L3-4 disc in 5\%, at the L-4 vertebral body in 43\%, at the L4-5 disc in $11 \%$, and at the $L-5$ vertebral body in $9 \%$. The confluence of the iliac veins was found at lower levels: $45 \%$ at the L-4 level, $19.39 \%$ at the L4-5 intervertebral disc, and $34 \%$ at the L-5 vertebral body.

CONCLUSIONS An oblique corridor of access to the L2-5 discs was found in $90 \%$ of the MR images $(99 \%$ access to $L 2-3,100 \%$ access to $L 3-4$, and $91 \%$ access to $L 4-5)$. Access to the L5-S1 disc was also established in $69 \%$ of the MR images analyzed. The lower the confluence of iliac veins, the less probable it was that access to the L5-S1 intervertebral disc space was observed. These findings support the use of lumbar MRI as a tool to predetermine the presence of an oblique corridor for access to the L2-S1 intervertebral disc spaces prior to lumbar spine surgery.

http://thejns.org/doi/abs/10.3171/2015.3.SPINE13976

KEY WORDS oblique lumbar interbody fusion; lumbar fusion; minimally invasive surgical approach; magnetic resonance imaging; retroperitoneal; oblique corridor

$\mathrm{M}$ INIMALLY invasive surgical (MIS) approaches for spine surgery have been popularized in recent years. New, less-invasive surgical techniques have evolved, becoming the preferred choice of many surgeons as a relatively safer alternative to traditional open surgery. ${ }^{1,23,27,33}$ Anterior, transforaminal, lateral transpsoas, transsacral, and oblique MIS approaches are used for intervertebral disc access. Each of these approaches carries its own risks and benefits. ${ }^{33}$ Studies have shown that MIS approaches lead to less tissue damage and faster recovery. ${ }^{1,33}$ Other techniques, such as laparoscopic interventions, have also been proposed. However, these techniques have limited use due to technical complexity, steep learning curves, and lack of improved morbidity. ${ }^{34}$

Since the introduction of MIS approaches, the morbidity related to the lumbar interbody fusion has significantly decreased. However, there are potential risks associated with each approach. ${ }^{33}$ The avoidance of vascular, visceral,

ABBREVIATION MIS = minimally invasive surgical. 
and sexual complications, as well as paraspinal denervation and dural tear, is among the reasons the lateral transpsoas approach is often preferred over other minimally invasive techniques. ${ }^{17}$ Conversely, lesion of the lumbar plexus or femoral nerve as they course through the psoas is a potential risk of the lateral transpsoas approach. ${ }^{15,18,19}$ An extremely variable incidence of sensory $(1 \%-75 \%)$ and motor $(0 \%-30 \%)$ complications in the ipsilateral (nondependent) lower extremity has been documented in transpsoas clinical publications. ${ }^{1,2,4,6,12,13,20-22,25,29,31,32,35,36,39}$ Muscle and nerve injury, either independently or in combination, are suspected to play a role in these postoperative complications.

Despite these reported complication rates, the transpsoas approach has continued to be the preferred method for many surgeons, due to the size and shape of intervertebral spacer that can be placed into the disc space. The surface area and span of these spacers are considered to be superior and therefore worth the risk of neural complications. ${ }^{2}$ The MIS oblique approach to the lumbar spine while the patient is in a lateral decubitus position has been proposed as an alternative method of inserting the same type and shape of intervertebral spacers, while mitigating the risk to the lumbar plexus that is inherent during the transpsoas approach. This theory makes sense with regard to the anatomical location of the lumbar plexus, but clinical outcomes of the MIS oblique technique are yet to be published.

A previous gross anatomy publication documented the oblique corridor L2-S1 intervertebral disc space in 21 cadavers. This cadaveric study found the corridor to be present in $100 \%$ of the specimens. To our knowledge, the present study is the first to analyze the existence of an oblique corridor to the lumbar spine based on MRI studies. The purpose of this study was to document the oblique corridor through MR image analysis, thus validating MRI as a means of presurgical planning for oblique lumbar interbody fusion.

\section{Methods}

One hundred thirty-three MR images of the lumbar spine obtained between May 4, 2012, and February 27, 2013, were randomly selected from our images database. Exclusion criteria consisted of previous lumbar or retroperitoneal surgery; there were no other exclusion criteria. One hundred thirty-three MR images were reviewed to achieve our goal of including 100 in this study. Thirtythree scans (24.8\%) were excluded: $15(11.3 \%)$ due to the absence of a coronal view, 7 (5.3\%) for technical issues (e.g., subject motion during scan, digital image error), and $11(8.3 \%)$ because of anatomical alteration related to prior lumbar surgery. One hundred MR images were included in the study; $47 \%$ of subjects were women and $53 \%$ were men. The average age was 57.4 years (range 22-88 years). Diagnoses of record related to the MRI included lumbago, lumbar stenosis, lumbar spondylosis, lumbar degenerative disc disease, sciatica, and lumbar radiculitis.

The MR images were first reviewed by a doctor of medicine research intern. The scans were then reviewed by 2 (physical medicine and rehabilitation) spine special- ists. T2-weighted MR images were analyzed in 3 different planes: coronal, axial, and sagittal. The L2-3, L3-4, and L4-5 intervertebral discs were first localized in the axial view. The oblique corridors were defined as the distance between the lateral border of the aorta (or the nearest common iliac vessel if below the aortic bifurcation) and the anterior medial border of the psoas muscle (Fig. 1). At the L5-S1 level, 2 measurements were taken. The coronal view was used as a reference to identify the first vessel that crossed the midline below the aortic bifurcation. The distance from this vessel to the inferior endplate of L-5 was measured vertically in the midsagittal plane (Fig. 2). In addition, a transverse measurement was taken from the midsagittal plane at the inferior endplate of L-5 to the medial border of the left common iliac vessel (most often the left common iliac vein) in the axial view (Fig. 3). The aforementioned measurements combine to define the L5-S1 oblique corridor. In some cases, the common iliac vessels were found blocking the oblique corridor to the L5-S1 disc. In these MR images, the distance between the psoas and the iliac vessels was measured as an alternative route to access the L5-S1 disc.

\section{Statistical Analysis}

Descriptive statistics were used to characterize the oblique corridor to the L2-S1 discs. The Student t-test was used to compare the differences between levels and between sexes. Chi-square analysis was used to find a statistical difference in the level of the aortic bifurcation and iliocaval junction between men and women. All statistical analyses were done using SAS statistical software (SAS Institute, Inc.).

\section{Results}

The oblique corridors to the L2-S1 discs were found between the psoas and the aorta or the left common iliac artery in $90 \%$ of the studied MR images. In 1 MR image, a large psoas muscle was in direct contact with the

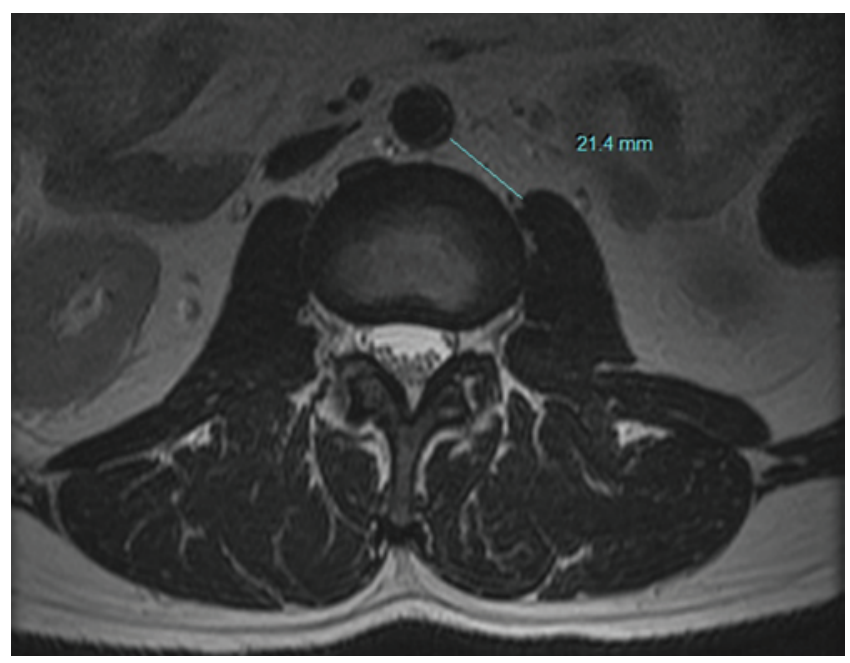

FIG. 1. Axial MR image with measurement of the oblique corridor. Figure is available in color online only. 


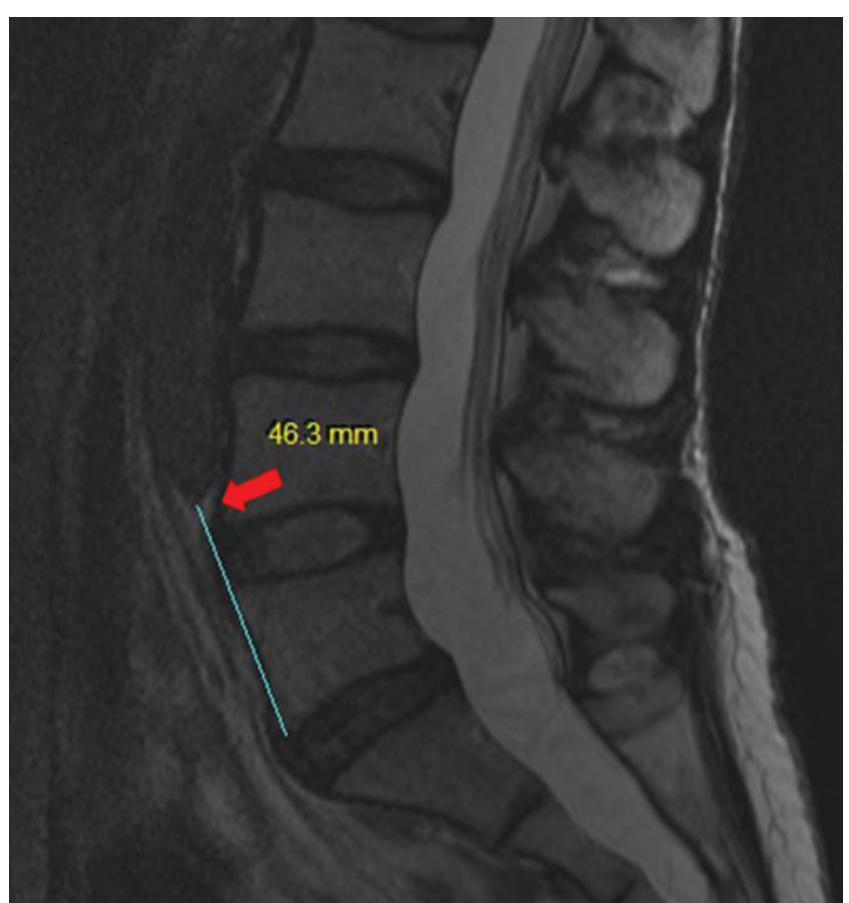

FIG. 2. Sagittal MR image with measurement of the distance from the first vessel crossing midline to the inferior endplate of $L-5$. Figure is available in color online only.

lateral border of the aorta, blocking access to the L2-3 intervertebral disc. All MR images had a clear corridor to the L3-4 disc. At the L4-5 level, 9 (9\%) of the studied MR images displayed no space between the aorta and the psoas, whereas the oblique corridor to the L5-S1 disc was observed in $69 \%$ of cases. Only 1 of the MR images did not have access to more than 1 level (L4-5 and L5-S1). Table 1 reports the statistics obtained from these measurements.

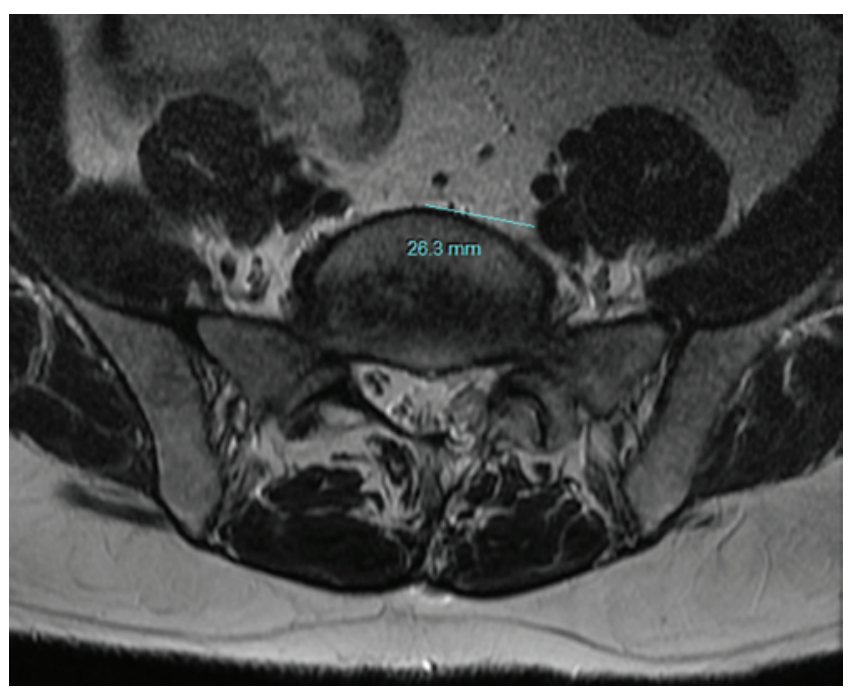

FIG. 3. Axial MR image with transverse measurement of the inferior endplate of $\mathrm{L}-5$ to the left common iliac vessel. Figure is available in color online only.
TABLE 1. Oblique corridor statistics

\begin{tabular}{lclll}
\hline \multicolumn{1}{c}{ Disc Level } & $\begin{array}{c}\text { Mean } \\
\text { Distance, } \mathrm{mm}\end{array}$ & $\mathrm{SD}$ & $\begin{array}{c}\text { Min, } \\
\mathrm{mm}\end{array}$ & $\begin{array}{c}\text { Max } \\
\mathrm{mm}\end{array}$ \\
\hline L2-3 & 16.04 & 5.83 & 0 & 32.9 \\
\hline L3-4 & 14.21 & 5.7 & 2.8 & 35.5 \\
\hline L4-5 & 10.28 & 6.81 & 0 & 26.2 \\
\hline L5-S1 transverse & 10 & 8.3 & 0 & 28.4 \\
\hline L5-S1 vertical & 10.13 & 9.6 & 0 & 49.9 \\
\hline Oblique access to L-5* & 10.8 & 9.2 & 4.6 & 24.6 \\
\hline
\end{tabular}

Max = maximum; $\min =$ minimum.

* In $31 \mathrm{MR}$ images (31\%), no anterior access to the inferior endplate of L-5 was observed. However, in 4 (12.9\%) of these 31 MR images, an oblique access to $L-5$ was found between the psoas muscle and iliac artery.

The size of the oblique corridors to the L2-5 discs was compared between men and women to determine whether sex can influence the dimension of the corridors. The access windows to the L2-3 and L3-4 discs were found to be slightly larger in men than women. In contrast, larger access windows to the L4-5 and L5-S1 discs were observed in women. However, when using a chi-square statistical analysis to compare the difference between sexes, no statistically significant differences were found (Table 2). These data show that despite the anatomical difference between men and women, the sex of the patient will not significantly influence the size of the corridor.

The oblique corridor was found to be largest at L2-3 and subsequently decreased in size at each lower level. The Student t-test was used to compare the oblique corridor at each level from L-2 to L-5. Statistically significant differences were found between all levels analyzed (Table 3).

The aortic bifurcation was observed between the lower third of the L-3 vertebral body and the middle third of the L-5 vertebral body. Meanwhile, the confluence of common iliac veins was found between the superior third of the L-4 vertebral body and the inferior third of the L-5 vertebral body. Chi-square analysis was used to compare the level of the aortic bifurcations and iliocaval junction in relation to the sex of the subjects. Although differences were observed between the sexes, they were not statistically significant (iliocaval junction $\mathrm{p}$ value $=0.086$, aortic bifurcation $\mathrm{p}$ value $=0.85$ ) (Table 4 ).

A relationship was found between the confluence of common iliac veins and anterior access to the L5-S1 disc. The lower the confluence, the less probable it was that anterior access to the L5-S1 disc was found. In 25 of 34 cases $(73 \%)$ where the iliocaval junction was identified at the L-5 level, no access to the L5-S1 disc was observed (Table 5). This outcome makes the level of confluence of the common iliac veins a determining factor to predict accessibility to the L5-S1 disc. Interestingly, in 3 of 8 cases (38\%) where the iliocaval junction was found at the middle third of the L-5 vertebral body, oblique access to the L5-S1 disc was found. In addition, oblique access to the L5-S1 disc was also found in a sole case where the iliocaval junction was identified in the inferior third of the L-5 vertebral body. 
TABLE 2. Oblique corridor analysis by sex

\begin{tabular}{|c|c|c|c|c|c|c|c|c|c|}
\hline \multirow[b]{2}{*}{ Disc Level } & \multicolumn{2}{|c|}{ Mean Distance, $\mathrm{mm}$} & \multicolumn{2}{|c|}{ SD } & \multicolumn{2}{|c|}{ Min, $\mathrm{mm}$} & \multicolumn{2}{|c|}{ Max, mm } & \multirow{2}{*}{$\begin{array}{l}p \text { Value } \\
\text { (M vs F) }\end{array}$} \\
\hline & M & $\mathrm{F}$ & M & $\mathrm{F}$ & M & $\mathrm{F}$ & M & $\mathrm{F}$ & \\
\hline L2-3 & 16.5 & 15.51 & 6.44 & 5.07 & 2.8 & 0 & 32.9 & 26 & 0.4005 \\
\hline L3-4 & 14.51 & 13.8 & 6.47 & 4.7 & 2.8 & 4.5 & 35.5 & 25.3 & 0.5826 \\
\hline L4-5 & 9.15 & 11.54 & 6.79 & 6.6 & 0 & 0 & 23.8 & 26.2 & 0.0808 \\
\hline L5-S1 transverse & 9.13 & 11.17 & 7.92 & 8.7 & 0 & 0 & 27.5 & 28.4 & 0.2231 \\
\hline L5-S1 vertical & 9.41 & 10.94 & 9.33 & 9.95 & 0 & 0 & 49.9 & 48.6 & 0.4322 \\
\hline Oblique access to $L-5^{*}$ & 10.8 & NA & 9.22 & NA & 4.6 & NA & 24.6 & NA & NA \\
\hline
\end{tabular}

$\mathrm{NA}=$ not applicable.

* In $31 \mathrm{MR}$ images (31\%), no anterior access to the inferior endplate of L-5 was observed. However, in 4 (12.9\%) of these 31 MR images, an oblique access to L- 5 was found between the psoas muscle and iliac artery.

\section{Anatomical Variants}

Two cases of immobile S1-2 discs were found. One of these subjects had an open oblique corridor at L2-3, L3-4, L4-5, and L5-S1, whereas the other subject had no oblique corridor at the L4-5 disc space. Four cases of Castellvi Class $\mathrm{IB}^{8}$ transitional anatomy were identified. The largest oblique access corridor to the L5-S1 disc (48.6 mm vertical and $28.4 \mathrm{~mm}$ transverse) was found in 1 of the transitional anatomy subjects. Transitional anatomy does not appear to negatively impact the oblique corridor to the L2-S1 discs. In some cases, it may facilitate access to the L5-S1 disc. However, further studies and larger sample sizes will be needed to validate this observation.

\section{Discussion}

As detailed in several publications, ${ }^{1,23,33}$ lumbar interbody fusion has been used for the treatment of spinal disorders since the 1930s, when Capener ${ }^{7}$ described the anterior approach for the treatment of spondylolisthesis. However, due to the high incidence of vascular injuries, the use of this technique was limited. ${ }^{1}$ In 1953, Cloward described lumbar interbody fusion performed through a posterior laminectomy, with the aim of preserving the facet joints and reducing the risk of vascular injuries. ${ }^{11}$ The open transforaminal approaches, which are a modification of the posterior approach, were also used for those purposes. These procedures required nerve root retraction, which often translated into postoperative neurological compromise. ${ }^{1}$ In addition to the complications associated with each approach, the open techniques also carried higher risks of infection, postoperative pain, and abdominal wall defects, as well as longer surgery and recovery times., ${ }^{1,19,37}$

As technology has evolved, techniques have improved, with a focus on tissue-sparing MIS approaches in an at-

TABLE 3. Oblique corridor measurement difference between levels, all subjects

\begin{tabular}{cr}
\hline Levels & p Value \\
\hline L2-3 vs L3-4 & 0.0005 \\
\hline L2-3 vs L4-5 & $<0.0001$ \\
\hline L3-4 vs L4-5 & $<0.0001$ \\
\hline
\end{tabular}

tempt to decrease the morbidity associated with a given surgery. The first case of laparoscopic anterior lumbar spine surgery, published in 1991 by Obenchain, was one of the first minimally invasive spine surgery procedures. ${ }^{28}$ However, the use of laparoscopic approaches was limited by the high incidence of large vessel and nerve injuries associated with the procedures. ${ }^{25,37}$ In recent years, minimally invasive approaches have become a safe alternative to open surgeries. The smaller incision and shorter operative and recovery times often result in less morbidity. ${ }^{11,19,25}$ Anterior, transforaminal, lateral transpsoas, transsacral, and oblique MIS approaches have been described to access the intervertebral discs and the anterior spine.

In 2011, Pimenta et al. first reported a modification of the anterior technique, described as a minimally invasive, extreme lateral approach for total disc replacement. ${ }^{29}$ This approach is now preferred by many surgeons. ${ }^{1,23}$ The small incision required for this procedure results in a shorter recovery time. Conversely, this approach has unique risks, such as lower-extremity motor and sensory deficits. Psoas disruption and its consequent inflammation is considered a possible cause of these postoperative symptoms. ${ }^{38}$ Injury of the lumbar plexus as it courses through the psoas muscle is another potential source of postoperative neurological symptoms. ${ }^{3,13-15}$ Intraoperative neurological monitoring is said to decrease neural injury. The NeuroVision JJB System (NuVasive) received FDA approval in 2001 for use in the operating room and noncritical care clinical environment for neurological monitoring and status assessment. ${ }^{5,13,14,18}$ However, Houten et al. described 2 cases where a lateral transpsoas approach was used with intraoperative neurological monitoring that still resulted in L-4 nerve root injury. ${ }^{18}$

Watkins originally described an open approach to the oblique corridor for access to the intervertebral disc space in his textbook in $1983 .{ }^{40}$ Mayer described an MIS oblique approach in $1997 .{ }^{24}$ Due to the incidence of neurological complications following the transpsoas approach, there has been a renewed interest in an MIS approach to the oblique corridor of the lumbar spine. In 2014, Davis et al. defined the oblique corridor in a cadaveric study with specimens in the right lateral decubitus position..$^{16}$ Davis et al. described an oblique retroperitoneal corridor to the intervertebral discs at L2-5 as well as L5-S1.

This oblique approach can be used to implant an in- 
TABLE 4. Aortic bifurcation and iliocaval junction by sex

\begin{tabular}{|c|c|c|c|c|c|c|c|c|c|}
\hline & & \multicolumn{4}{|c|}{ Aortic Bifurcation } & \multicolumn{4}{|c|}{ Confluence of Common lliac Veins } \\
\hline & & \multicolumn{2}{|c|}{ Frequency } & \multicolumn{2}{|c|}{$\%$} & \multicolumn{2}{|c|}{ Frequency } & \multicolumn{2}{|c|}{$\%$} \\
\hline \multicolumn{2}{|c|}{ Disc Level } & M & $\mathrm{F}$ & M & $\mathrm{F}$ & M & $\mathrm{F}$ & M & $\mathrm{F}$ \\
\hline L-3 vertebral body & Inferior third & 2 & NA & 3.78 & NA & NA & NA & NA & NA \\
\hline L3-4 disc & & 2 & 3 & 3.78 & 6.38 & NA & NA & NA & NA \\
\hline \multirow[t]{3}{*}{ L-4 vertebral body } & Superior third & 4 & 4 & 7.55 & 8.52 & 1 & NA & 1.92 & NA \\
\hline & Middle third & 18 & 15 & 33.95 & 31.91 & 7 & 11 & 13.46 & 23.91 \\
\hline & Inferior third & 15 & 17 & 28.30 & 36.17 & 11 & 15 & 21.16 & 32.61 \\
\hline L4-5 disc & & 6 & 5 & 11.32 & 10.64 & 15 & 4 & 28.85 & 8.70 \\
\hline \multirow[t]{3}{*}{ L-5 vertebral body } & Superior third & 4 & 3 & 7.55 & 6.38 & 13 & 12 & 25.00 & 26.08 \\
\hline & Middle third & 2 & NA & 3.78 & NA & 4 & 4 & 7.69 & 8.70 \\
\hline & Inferior third & NA & NA & NA & NA & 1 & NA & 1.92 & NA \\
\hline Total & & 53 & 47 & $100.01^{*}$ & 100.00 & $52 \dagger$ & $46 \dagger$ & 100.00 & 100.00 \\
\hline
\end{tabular}

* Values do not add to $100 \%$ due to rounding.

$\dagger$ In 2 of the reviewed MR images, the vena cava bifurcation was not observed. In 1 case, the 2 common iliac veins run lateral along the aorta and no vena cava is observed. In the second case, the abdominal vena cava is identified; however, there is no bifurcation observed. Entering the pelvis, the vena cava lateralized to the right side.

terbody spacer similar in shape to that placed during a transpsoas approach. Intraoperatively, the patient is placed in a lateral decubitus position and the surgeon stands facing the patient's ventral side, accessing the disc space from an anterior oblique angle. With mild finger and instrument retraction of the psoas and abdominal structures, sequential tubular dilating retractors are placed through the oblique corridor. These retractors stay within the oblique corridor, lateral to the vascular structures and medial to the psoas (Fig. 4).

When compared with the direct lateral approach, the change in trajectory is angular rather than an anterior translation (Fig. 5). The vertex of the surgical trajectory is at the center of the targeted intervertebral disc space. The retractors are then placed along this trajectory through the oblique corridor. Tubular retractors can be secured either to the table via fixation arms or directly to the vertebral body above and below the disc space. A complete discectomy and implant placement can be completed through the tubular retractors. Using this approach, the ureter, peritoneum, and major vessels are often out of the surgical field. If these structures are encountered, they can be visualized directly and retracted toward midline. ${ }^{16}$ Our MRI

\section{TABLE 5. Anterior access to L5-S1 related to the level of the iliocaval junction}

\begin{tabular}{lcc}
\hline $\begin{array}{c}\text { Iliocaval Junction } \\
\text { Level }\end{array}$ & $\begin{array}{c}\text { No. of } \\
\text { Subjects }\end{array}$ & $\begin{array}{c}\text { No. of Subjects w/ No Anterior } \\
\text { Access to L5-S1 (\%)* }\end{array}$ \\
\hline Inferior third of L-4 & 18 & $2(11)$ \\
\hline L4-5 disc & 19 & $4(21)$ \\
\hline Superior third of L-5 & 25 & $16(64)$ \\
\hline Middle third of L-5 & 8 & $8(100)$ \\
\hline Inferior third of L-5 & 1 & $1(100)$ \\
\hline
\end{tabular}

${ }^{*}$ Expressed as a percentage relative to the number of subjects for each level. study describes this same oblique corridor to the L2-S1 disc spaces, as seen on 100 MRI studies.

Access to the L2-3 and L3-4 discs was found in 99\% and $100 \%$ of cases, respectively. The largest oblique cor-

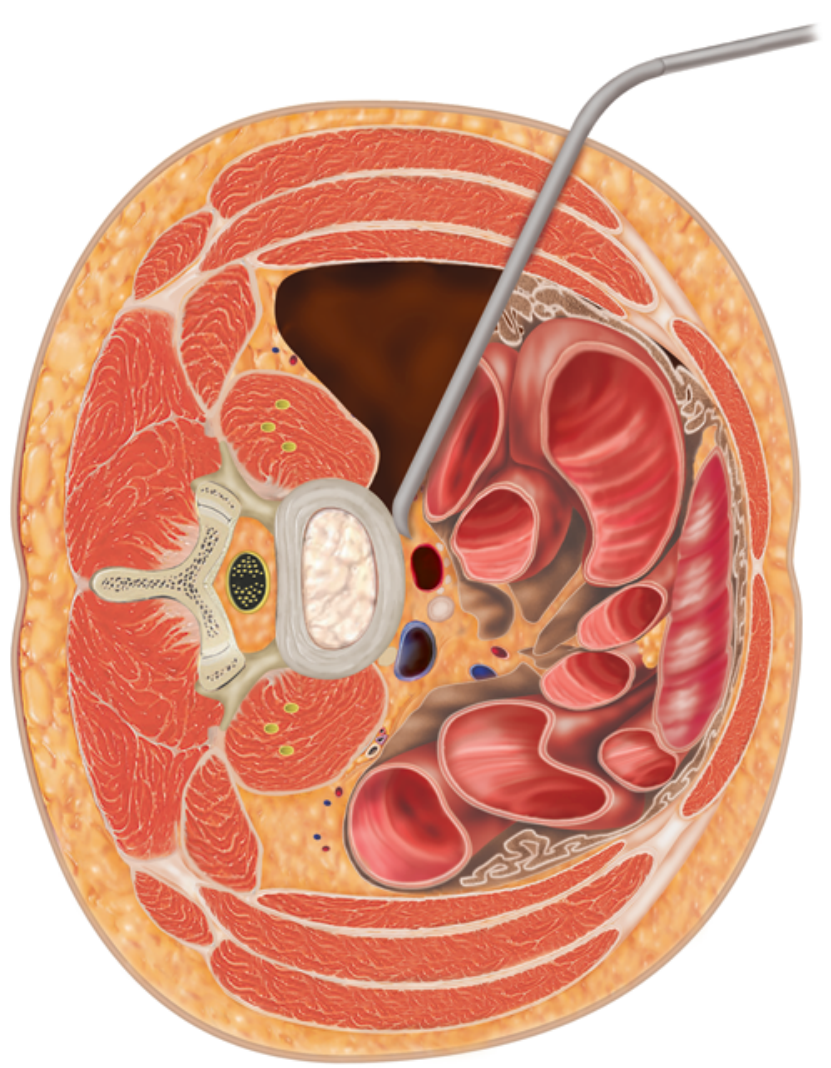

FIG. 4. Illustration showing the oblique lumbar interbody fusion technique: retraction of the abdominal viscera in preparation for placement of tubular retractors. Image provided by Medtronic, Inc., and published with permission. Figure is available in color online only. 


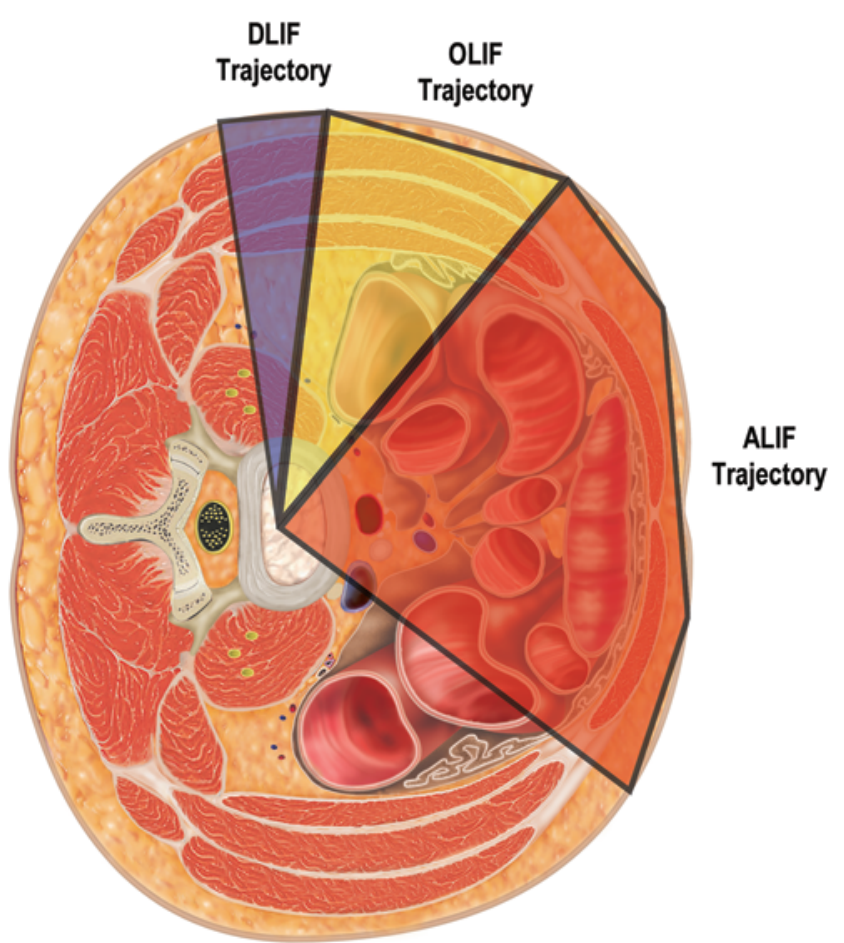

FIG. 5. Illustration showing the trajectory of an anterior versus lateral versus oblique approach to the lumbar disc space. ALIF = anterior lumbar interbody fusion; DLIF = direct lateral interbody fusion; OLIF = oblique lumbar interbody fusion. Image provided by Medtronic, Inc., and published with permission. Figure is available in color online only.

ridor was observed at the L2-3 level and became progressively smaller in the lower levels (L3-4: $14.2 \mathrm{~mm}$; L4-5: $10.28 \mathrm{~mm}$; L5-S1: transverse measurement $10 \mathrm{~mm}$, vertical measurement $10.13 \mathrm{~mm}$ ). Statistically significant differences were found when all levels were compared; however, no significant difference was found when they were compared between sexes. These data show that despite the anatomical differences between men and women, sex should not be considered a determining factor of predicting the size of the oblique corridors. At the L5-S1 level, the abdominal vessels were found blocking anterior access to the disc in $31 \%$ of cases. The proximity of the psoas to the iliac vascular structures also prevented an oblique access to the disc. However, in 4 of $31 \mathrm{MR}$ images with no anterior access to the L5-S1 disc, an oblique access between the psoas and the inguinal vessels was observed. This approach could be used as an alternative for those patients for whom anterior access to L5-S1 is obstructed by the lower abdominal vessels. The anatomy of the abdominal vessels, especially the confluence of the common iliac veins, was the most determining factor for L5-S1 disc access. The lower the vena cava bifurcation, the less probable it was that access to the L5-S1 disc was observed. During this study, the bifurcation of the aorta was found between the lower third of the L-3 vertebral body and the middle third of the L-5 vertebral body. Meanwhile, the confluence of the common iliac veins was found between the superior third of the L- 4 vertebral body and the inferior third of the L-5 vertebral body.

The anatomy of the abdominal vessels and its relationship to the lumbar spine has been previously studied by Moussallem et al. ${ }^{26}$ using CT, and Pirró et al. ${ }^{30}$ using cadaveric dissection. A larger study by Chithriki et al. in $2002,{ }^{10}$ using MRI, reported the aortic bifurcation between the superior third of the L-3 vertebral body and the middle third of the L-5 vertebral body, which is slightly higher compared with our study (i.e., inferior third of L-3). Most of the aortic bifurcations were observed at the L-4 vertebral level in both studies $(66.9 \%$ in the study by Chithriki et al. and $73 \%$ in the present study). There was some variation in the exact level as it relates to which third of the L-4 vertebral body, but these differences are probably due to normal anatomical variation. In a review of 30 MR images, Vaccaro et al. ${ }^{38}$ reported $73 \%$ of cases with an aortic bifurcation at L-4 and $57 \%$ of cases with a confluence of the common iliac veins at L-5. Our observations of the common iliac vein confluence had slightly more variation, with $34.67 \%$ at the L-5 vertebral body level, $45.92 \%$ at L-4, and $19.39 \%$ at the L4-5 disc level. In a study of 124 subjects by Capellades et al., ${ }^{9}$ the confluence of common iliac veins was found between the lower third of L-4 and the superior third of L-5 in $59.4 \%$ of subjects.

Oblique access to the lumbar spine has also been studied through cadaveric dissection. ${ }^{16}$ In a cadaveric study by Davis et al., the distance between the abdominal vessels and the psoas was measured after cadaveric dissection. ${ }^{16}$ Clear access to each of the L2-S1 disc spaces was found in all specimens. These corridors were larger (with and without psoas retraction) when compared with the findings in this study (Table 6). ${ }^{25}$ Differences in positioning (lateral decubitus during dissection and supine during MRI) may account for some of these differences. Although subjects were not in the operative position, the present study supports lumbar MRI as a good predictor of the oblique corridor of access to the $\mathrm{L} 2-\mathrm{S} 1$ discs. Psoas retraction and a lateral decubitus position are believed to increase the sizes of the corridors. Common iliac vascular retraction at L5-

TABLE 6. Oblique corridor morphological study versus MRI study

\begin{tabular}{lccccc}
\hline \multicolumn{1}{c}{ Type of Study } & $\begin{array}{c}\text { L2-3 Mean } \\
\text { Distance, } \mathrm{mm}\end{array}$ & $\begin{array}{c}\text { L3-4 Mean } \\
\text { Distance, } \mathrm{mm}\end{array}$ & $\begin{array}{c}\text { L4-5 Mean } \\
\text { Distance, } \mathrm{mm}\end{array}$ & $\begin{array}{c}\text { L5-S1 Transverse } \\
\text { Mean Distance, mm }\end{array}$ & $\begin{array}{c}\text { L5-S1 Vertical } \\
\text { Mean Distance, mm }\end{array}$ \\
\hline Cadaveric dissection* $^{*}$ & 18.60 & 19.25 & 15.00 & 14.75 & 23.85 \\
\hline Cadaveric dissection-psoas retraction* & 25.50 & 27.05 & 24.45 & NA & 10 \\
\hline MRI & 16.04 & 14.21 & 10.28 & 10 & 10.13 \\
\hline
\end{tabular}

* According to the study by Moller et al. 
S1 would probably allow access to most of the subjects in this study, very similar to the method of a standard anterior lumbar interbody fusion approach.

Further studies should be performed for better clinical correlation. Dimensions of the oblique corridor in preoperative MR images should be directly compared with those obtained during oblique lumbar interbody fusion on the same subject. These results would be useful to overcome some limitations encountered during this study, which included positioning of the subject during MRI versus that during surgery. The current study does not have enough information to conclude that oblique lumbar interbody fusion is superior to surgical techniques currently used.

\section{Conclusions}

The oblique approach for lumbar interbody fusions has been suggested as an alternative to the transpsoas approach to the L2-5 intervertebral disc space, while keeping the patient in the lateral decubitus surgical position. This oblique approach has also been suggested to access the L5-S1 disc while maintaining the lateral decubitus position. Benefits of the oblique approach include avoiding the psoas muscle and lumbar plexus; negating the need for a jack-knife position, which has been postulated to cause neuropraxia of the lumbar plexus on the nondependent lower extremity; allowing access to the L5-S1 disc space without repositioning; and allowing the use of large lateral interbody cage placement similar to the transpsoas approach.

This study documents that $90 \%$ of the MR images reviewed showed oblique access to each of the L2-5 intervertebral discs between the psoas and abdominal vessels. Ninety-nine percent of the MR images had oblique access to the L2-3 disc, $100 \%$ had access to the L3-4 disc, and $91 \%$ to the L4-5 disc. The anatomy of the abdominal vessels limited anterior access to the L5-S1 disc, and only $69 \%$ had clear access. A relationship between the vena cava bifurcation and access to the L5-S1 disc was observed: the lower the bifurcation of the vena cava, the less probable it was that access to the L5-S1 disc was found. Sex did not account for any differences in oblique corridor measurements. L2-3 had the largest oblique corridor measurements, with the size of the oblique corridors decreasing at each lower level. In conclusion, this study supports using MRI for preoperative planning prior to an MIS oblique retroperitoneal approach for lumbar interbody fusion.

\section{References}

1. Anand N, Rosemann R, Khalsa B, Baron EM: Mid-term to long-term clinical and functional outcomes of minimally invasive correction and fusion for adults with scoliosis. Neurosurg Focus 28(3):E6, 2010

2. Baron EM, Davis T, Anand N: The transpsoas approach for thoracolumbar interbody fusion, in Vaccaro AR, Baron EM (eds): Spine Surgery: Operative Techniques. Philadelphia: Elsevier Saunders, 2012, pp 314-326

3. Benglis DM, Vanni S, Levi AD: An anatomical study of the lumbosacral plexus as related to the minimally invasive transpsoas approach to the lumbar spine. J Neurosurg Spine 10:139-144, 2009
4. Berjano P, Balsano M, Buric J, Petruzzi M, Lamartina C: Direct lateral access lumbar and thoracolumbar fusion: preliminary results. Eur Spine J 21 (Suppl 1):S37-S42, 2012

5. Brau SA, Delamarter RB, Kropf MA, Watkins RG III, Williams LA, Schiffman ML, et al: Access strategies for revision in anterior lumbar surgery. Spine (Phila Pa 1976) 33:16621667,2008

6. Cahill KS, Martinez JL, Wang MY, Vanni S, Levi AD: Motor nerve injuries following the minimally invasive lateral transpsoas approach. J Neurosurg Spine 17:227-231, 2012

7. Capener N: Spondylolisthesis. Br J Surg 19:374-386, 1932

8. Castellvi AE, Goldstein LA, Chan DP: Lumbosacral transitional vertebrae and their relationship with lumbar extradural defects. Spine (Phila Pa 1976) 9:493-495, 1984

9. Capellades J, Pellisé F, Rovira A, Grivé E, Pedraza S, Villanueva C: Magnetic resonance anatomic study of iliocava junction and left iliac vein positions related to L5-S1 disc. Spine (Phila Pa 1976) 25:1695-1700, 2000

10. Chithriki M, Jaibaji M, Steele RD: The anatomical relationship of the aortic bifurcation to the lumbar vertebrae: a MRI study. Surg Radiol Anat 24:308-312, 2002

11. Cloward RB: The treatment of ruptured lumbar intervertebral discs by vertebral body fusion. I. Indications, operative technique, after care. J Neurosurg 10:154-168, 1953

12. Cummock MD, Vanni S, Levi AD, Yu Y, Wang MY: An analysis of postoperative thigh symptoms after minimally invasive transpsoas lumbar interbody fusion. J Neurosurg Spine 15:11-18, 2011

13. Dakwar E, Cardona RF, Smith DA, Uribe JS: Early outcomes and safety of the minimally invasive, lateral retroperitoneal transpsoas approach for adult degenerative scoliosis. Neurosurg Focus 28(3):E8, 2010

14. Dakwar E, Vale FL, Uribe JS: Trajectory of the main sensory and motor branches of the lumbar plexus outside the psoas muscle related to the lateral retroperitoneal transpsoas approach. J Neurosurg Spine 14:290-295, 2011

15. Davis TT, Bae HW, Mok JM, Rasouli A, Delamarter RB: Lumbar plexus anatomy within the psoas muscle: implications for the transpsoas lateral approach to the L4-L5 disc. J Bone Joint Surg Am 93:1482-1487, 2011

16. Davis TT, Hynes RA, Fung DA, Spann SW, MacMillan M, Kwon B, et al: Retroperitoneal oblique corridor to the L2-S1 intervertebral discs in the lateral position: an anatomic study. J Neurosurg Spine 21:785-793, 2014

17. Guérin P, Obeid I, Gille O, Bourghli A, Luc S, Pointillart V, et al: Safe working zones using the minimally invasive lateral retroperitoneal transpsoas approach: a morphometric study. Surg Radiol Anat 33:665-671, 2011

18. Houten JK, Alexandre LC, Nasser R, Wollowick AL: Nerve injury during the transpsoas approach for lumbar fusion. J Neurosurg Spine 15:280-284, 2011

19. Hussain NS, Perez-Cruet MJ: Complication management with minimally invasive spine procedures. Neurosurg Focus 31(4):E2, 2011

20. Isaacs RE, Hyde J, Goodrich JA, Rodgers WB, Phillips FM: A prospective, nonrandomized, multicenter evaluation of extreme lateral interbody fusion for the treatment of adult degenerative scoliosis: perioperative outcomes and complications. Spine (Phila Pa 1976) 35 (26 Suppl):S322-S330, 2010

21. Kepler CK, Sharma AK, Huang RC: Lateral transpsoas interbody fusion (LTIF) with plate fixation and unilateral pedicle screws: a preliminary report. J Spinal Disord Tech 24:363-367, 2011

22. Knight RQ, Schwaegler P, Hanscom D, Roh J: Direct lateral lumbar interbody fusion for degenerative conditions: early complication profile. J Spinal Disord Tech 22:34-37, 2009

23. Le TV, Uribe JS: The minimally invasive retroperitoneal transpsoas approach, in Chung KJ (ed): Spine Surgery. Rijeka, Croatia: InTech, 2012 
24. Mayer HM: A new microsurgical technique for minimally invasive anterior lumbar interbody fusion. Spine (Phila Pa 1976) 22:691-700, 1997

25. Moller DJ, Slimack NP, Acosta FL Jr, Koski TR, Fessler RG, Liu JC: Minimally invasive lateral lumbar interbody fusion and transpsoas approach-related morbidity. Neurosurg Focus 31(4): $\mathrm{E} 4,2011$

26. Moussallem CD, Abou Hamad I, El-Yahchouchi CA, Moussallem MD, Arnalsteen DM, Mertl P, et al: Relationship of the lumbar lordosis angle to the abdominal aortic bifurcation and inferior vena cava confluence levels. Clin Anat 25:866871, 2012

27. Mundis GM, Akbarnia BA, Phillips FM: Adult deformity correction through minimally invasive lateral approach techniques. Spine (Phila Pa 1976) 35 (26 Suppl):S312-S321, 2010

28. Obenchain TG: Laparoscopic lumbar discectomy: case report. J Laparoendosc Surg 1:145-149, 1991

29. Pimenta L, Oliveira L, Schaffa T, Coutinho E, Marchi L: Lumbar total disc replacement from an extreme lateral approach: clinical experience with a minimum of 2 years' follow-up. J Neurosurg Spine 14:38-45, 2011

30. Pirró N, Ciampi D, Champsaur P, Di Marino V: The anatomical relationship of the iliocava junction to the lumbosacral spine and the aortic bifurcation. Surg Radiol Anat 27:137141, 2005

31. Pumberger M, Hughes AP, Huang RR, Sama AA, Cammisa FP, Girardi FP: Neurologic deficit following lateral lumbar interbody fusion. Eur Spine J 21:1192-1199, 2012

32. Sharma AK, Kepler CK, Girardi FP, Cammisa FP, Huang RC, Sama AA: Lateral lumbar interbody fusion: clinical and radiographic outcomes at 1 year: a preliminary report. J Spinal Disord Tech 24:242-250, 2011

33. Shen FH, Samartzis D, Khanna AJ, Anderson DG: Minimally invasive techniques for lumbar interbody fusions. Orthop Clin North Am 38:373-386, vi, 2007

34. Silvestre C, Mac-Thiong JM, Hilmi R, Roussouly P: Complications and morbidities of mini-open anterior retroperitoneal lumbar interbody fusion: Oblique lumbar interbody fusion in 179 patients. Asian Spine J 6:89-97, 2012

35. Tohmeh AG, Rodgers WB, Peterson MD: Dynamically evoked, discrete-threshold electromyography in the extreme lateral interbody fusion approach. J Neurosurg Spine 14:3137,2011

36. Tormenti MJ, Maserati MB, Bonfield CM, Okonkwo DO,
Kanter AS: Complications and radiographic correction in adult scoliosis following combined transpsoas extreme lateral interbody fusion and posterior pedicle screw instrumentation. Neurosurg Focus 28(3):E7, 2010

37. Uribe JS, Arredondo N, Dakwar E, Vale FL: Defining the safe working zones using the minimally invasive lateral retroperitoneal transpsoas approach: an anatomical study. J Neurosurg Spine 13:260-266, 2010

38. Vaccaro AR, Kepler CK, Rihn JA, Suzuki H, Ratliff JK, Harrop JS, et al: Anatomical relationships of the anterior blood vessels to the lower lumbar intervertebral discs: analysis based on magnetic resonance imaging of patients in the prone position. J Bone Joint Surg Am 94:1088-1094, 2012

39. Wang MY, Mummaneni PV: Minimally invasive surgery for thoracolumbar spinal deformity: initial clinical experience with clinical and radiographic outcomes. Neurosurg Focus 28(3):E9, 2010

40. Watkins RG: Surgical Approaches to the Spine. New York: Springer, 1983

\section{Disclosure}

Dr. Davis has received clinical or research support for this study (includes equipment or material) from Medtronic. He is a consultant for Medtronic, Stryker, St. Jude, and Vertiflex. He has ownership in Alpha Diagnostics, Paradigm Spine, and Small Bone Innovations.

\section{Author Contributions}

Conception and design: all authors. Acquisition of data: Molinares. Analysis and interpretation of data: Fung, Molinares. Drafting the article: Fung, Molinares. Critically revising the article: Fung, Davis. Reviewed submitted version of manuscript: all authors. Approved the final version of the manuscript on behalf of all authors: Fung. Statistical analysis: Molinares. Administrative/technical/material support: Fung, Davis. Study supervision: Fung, Davis.

\section{Correspondence}

Daniel A. Fung, Orthopedic Pain Specialists, 2811 Wilshire Blvd., Ste. 850, Santa Monica, CA 90403. email: dfungmd@ gmail.com. 